CASE REPORT

\title{
Postural hallucinations? An unusual presentation of anaemia
}

\author{
A J Field, D J Cottrell
}

Arch Dis Child 2005;90:1192-1193. doi: 10.1136/adc.2005.075994

The case of a 14 year old girl is reported, referred to Child Psychiatry with tearfulness and possible auditory hallucinations. Closer questioning revealed a history of low energy, sleeping excessively without refreshment, exertional dyspnoea, and poor growth. Psychologically, there was no evidence of low mood or negative cognitions despite the inexplicable tearfulness. Detailed enquiry revealed the "hallucinations" to be "whooshing" noises in her ears precipitated by standing. She was found to have a haemoglobin level of $55 \mathrm{~g} / \mathrm{l}$, attributed to a combination of poor diet and menorrhagia.

Periodic, anaemic, cerebral hypoxia could be proposed to be the root of most, if not all, of the symptoms, illustrating the importance of marrying physical and psychological history taking with suitable investigations for an eminently treatable condition.

A number of symptoms exist which can reflect the presence of either physical or psychiatric illness, or both-fatigue is a good example-and can lead diagnosticians down one path without fully excluding others. This case shows how easily this can occur, even when considering common conditions.

\section{CASE REPORT}

A 14 year old girl was referred to Child Psychiatry with a number of months of tearfulness and "hearing voices". There was a family history of psychotic illness, depression, and suicide in a maternal aunt.

She lived at home with both parents, and was the youngest of three daughters. There were no problems during pregnancy or delivery, and developmental milestones were achieved appropriately. There were no difficulties at school, academically, or socially. She had no medical history of note aside from briefly being investigated for failure to thrive by paediatricians when aged 18 months, though her size was attributed to familial small build.

In essence, her symptoms fell into three main categories. Firstly, she had been tearful for the last seven months, but without any accompanying anhedonia, low mood, poor self esteem, or depressive thinking.

Secondly, the noises she had been hearing consisted of "whooshing or whispering" sounds, occurring only when she stood up or rushed around, and resolving on resting. They came from within her head, and were present more when she was tired. There had at no stage been any intelligible voicesshe described once hearing a low pitched rumbling inside her head which she felt may have been a voice but knew not to be real or audible to others-but she told her GP she felt the whispering was "angry". Associated with these noises, she felt dizzy and short of breath, with a racing heart.
Thirdly, even before the tearfulness began, she had been feeling lethargic. She slept for long periods without feeling refreshed. On exertion, she rapidly became breathless, and the muscles in her legs ached. She had not suffered any fits, faints, or funny turns, though had feared she may lose consciousness when she felt dizzy.

Her only other complaint was of heavy periods. She was slight, pale, and tired looking. Her height and weight were matched on the 0.4 th centile.

While a psychiatric differential diagnosis for auditory hallucinations would include psychotic depression, or indeed schizophrenia, the symptoms in this case do not fit.

There was no abnormality of mood, enjoyment, or thought (such as ideas of guilt, hopelessness, or low self-esteem), nor biological symptoms of insomnia or poor appetite suggestive of depressive illness. True auditory hallucinations diagnostic of psychotic processes take the form of one or more voices, from an external source, talking in the second or, more conclusively, third person, possibly giving commands or running commentary, and with a perceived reality that may cause the sufferer to respond to them. They would be beyond the sufferer's control-not remedied easily by resting, for example.

Given this odd history, we wondered whether any investigations had been performed, at which point her mother recalled the GP saying the girl's "blood count" was low. After contacting the GP, we obtained a haemoglobin result from the previous month-of $55 \mathrm{~g} / \mathrm{l}$. Although treatment with ferrous sulphate had been initiated and a referral made to Paediatrics, this was surprisingly not mentioned in the subsequent "urgent" referral made to Child Psychiatrynor had anyone registered that no appointment had been received from the paediatricians until we liaised with them. When she was reviewed, a diagnosis of profound microcytic anaemia secondary to menorrhagia and inadequate diet was made, and treatment with the oral contraceptive pill broached.

We hypothesised that the "noises" were the result of intermittent cerebral hypoxia, due to profound anaemia and possible postural hypotension, followed by compensatory circulatory hyperdynamism, potentially exacerbated by an anxiety response to the onset of symptoms. At her last review, on treatment, all her "psychiatric" symptoms had resolved.

\section{DISCUSSION}

Little clinical evidence exists describing such symptoms in relation to anaemia in children-though they bear resemblance to those observed in adults with cerebral hypoxia of other causes, for example, an elderly man with anaemic anoxia secondary to chronically bleeding haemorrhoids. ${ }^{1}$ Had there been convincing hallucinatory symptoms, it should be borne in mind that children can have auditory hallucinations in the absence of psychotic illness. ${ }^{2}$ 
The essential learning point here is that psychiatric diagnoses are not solely made in the absence of physical illness, and vice versa. The presence of a family history of mental illness may have provided a "red herring", but the history, examination, and investigations had already provided the diagnosis, had the information been appraised more holistically.

\section{Authors' affiliations}

A J Field, D J Cottrell, Academic Unit of Child and Adolescent Mental Health, Leeds, UK

Competing interests: none declared
Correspondence to: Dr A J Field, Academic Unit of Child and Adolescent Mental Health, 12a Clarendon Road, Leeds, LS2 9NN, UK; annafield@doctors.org.uk

Accepted 26 July 2005

\section{REFERENCES}

1 Lishman WA. Endocrine diseases and metabolic disorders. In: Organic psychiatry. The psychological consequences of cerebral disorder, 3rd edn. Blackwell Science Ltd, 1998:546-7.

2 Vickers B, Garralda E. Hallucinations in nonpsychotic children. J Am Acad Child Adolesc Psychiatry 2000;39:12-13.

\section{IMAGES IN PAEDIATRICS}

\section{Superglue mistaken for eye drops}

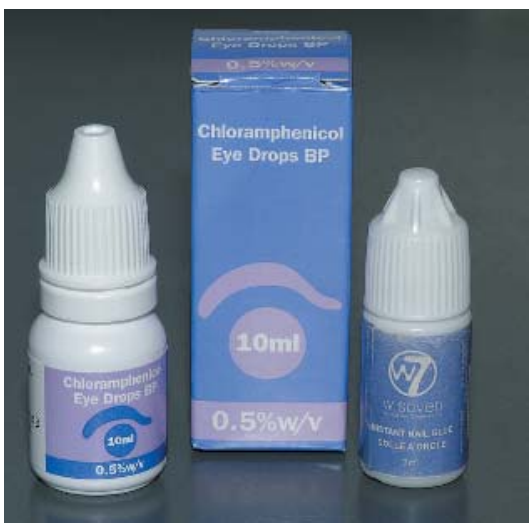

Figure 1 Packs of Chloramphenicol Eye Drops and Instant Nail Glue.

\begin{abstract}
yanoacrylate, better known as superglue, is sold as a "do it yourself" (DIY) pack. The shape and colour of the superglue bottle in these packs bears a striking resemblance to the container of commonly used eye drops (fig 1). McLean reported accidental ocular injury from it in 14 patients, of which five were children. ${ }^{1}$ We have recently seen three more cases in a short interval. In separate incidents children aged 7, 11, and 15 presented with ocular injuries (corneal abrasion and eyelid closure) due to superglue. All of them recovered with conservative treatment. No long term effects were seen.

These incidents have been reported with alarming regularity for the past two decades. ${ }^{2-4}$ Have we done enough to prevent them? We highlight the need for regulating non-pharmaceutical packaging. The nail glue container used in one of our cases had no obvious warning on the front and the print was quite small. The colour is also similar to that of the chloramphenicol eye drops bottle (fig 1). There are no design regulations on plastic bottles. ${ }^{5}$ As a result nonpharmaceutical companies are free to label and produce their products as they see fit. We highlight the need for the introduction of such regulation; for example, child resistant packaging, barring overseas language on bottles, and changing the size, shape, and colour of bottles.
\end{abstract}

S P Desai, B C Teggihalli, R Bhola Department of Ophthalmology, Doncaster Royal Infirmary, Armthorpe Road, Doncaster DN2 5LT, UK; desaisp@hotmail.com

Competing interests: none declared

\section{REFERENCES}

1 McLean CJ. Ocular superglue injury. J Accid Emerg Med 1997;14:40-1.

2 Lyons C, Stevens J, Bloom J. Superglue inadvertently used as eye drops. BMJ 1990;300:328.

3 Knight IJ. Mistaken eye drops and subsequent instillation of superglue. Eye 2001;15(pt 5):663.

4 Morgan SJ, Astbury NJ. Inadvertent self administration of superglue: a consumer hazard. BMJ 1984;289:226-7.

5 BSI. Specification of containers for pharmaceutical dispensing. Part 5. Eye drop bottles. London: British Standard Institute, 1973. 